\title{
The fate of dwarf satellite galaxies (or: Can we observe soft merging?)
}

\author{
G. Richter ${ }^{1}$, J. Vennik ${ }^{2}$, P. Böhm ${ }^{1}$ and M. Capaccioli ${ }^{3}$ \\ ${ }^{1}$ Astrophysikalisches Institut Potsdam, D-14482 Potsdam, Germany \\ ${ }^{2}$ Tartu Observatory, 61602 Tartumaa, Tõravere, Estonia \\ ${ }^{3}$ Osservatorio Astronomico di Capodimonte, via Moiariello 16, I-80131 \\ Napoli, Italy
}

\begin{abstract}
.
Dwarf satellite galaxies and globular clusters bound to luminous (in particular early type) galaxies, can be steered by interactions to orbits penetrating deep into the potential of their parent galaxy, where the outer stars are eroded and the dwarf satellite is completely destroyed in a relatively short time, resulting in very faint disturbances (soft merging) in the form of faint rings in the parent galaxy.

With special image processing methods we have found faint structures in $\mathrm{E}$ and $\mathrm{S} 0$ galaxies, which for many reasons (mass, statistics, etc.) can be interpreted as observational evidence of this type of soft merging. This debris of dwarf satellites can provide a considerable part of the mass of the parent galaxy.
\end{abstract}

\section{General background}

"Strong" Merging: It is well understood now - by theory, numerical simulations, and observations - that the encounter of massive galaxies results in a giant elliptical galaxy (e.g. Barnes 1992).

"Soft" Merging: There are a lot of arguments that strong merging is not the only, and perhaps not even the dominating process in galaxy evolution (Richter et al. 1997).

In particular: Since the bright galaxies are usually embedded in a cloud of satellites (Einasto et al. 1975) - dwarf galaxies and globular clusters - the interaction with other galaxies may continuously steer such dwarfs to orbits penetrating deep into the main galaxy.

The concept of "soft" merging means: Since the dwarf satellites are two to three orders of magnitude less massive than the main galaxy, the distortion of the structure of the main galaxy by a single event is only marginal. On the other hand, because of the large number of dwarfs, soft merging should be by far the most frequent type of merging. In galaxy clusters the satellites may be distributed as a general dwarf background. But this does not change essentially the soft merging phenomenon. 


\section{The mechanism of soft merging:}

Recent numerical simulations (e.g. Johnston et al. 1996) show that:

- Near perigalacticum, the outer stars of the satellite are "eroded".

- For centrum distances of the perigalacticum of a few kpc, the erosion is so efficient, that the satellite is essentially destroyed after a few Gyr.

- The debris of the erosion moves on rosetta orbits.

- Since every single star spends most of the time on the peripheral parts of its rosetta orbit (velocity $\sim \mathrm{r}^{-2}$ ), the whole debris yields essentially a ring.

- These rings are remarkably stable within time scales of order $10^{8}$ to $10^{9}$ years.

\section{Observational evidence}

"Moving star groups" in the Milky Way halo: Some teams (e.g. Johnston et al. 1996) are searching for the debris of satellites as moving groups of the halo stars in Hipparchos data set. Recently Helmi et al. (1999) found that $\sim 10 \%$ of metal-poor halo stars come from a single coherent structure that probably resembled a dwarf spheroidal galaxy.

Faint structures in Early Type Galaxies: The debris of soft merging can be expected to be seen as very faint distortions in the otherwise very smooth bodies of early type galaxies (late types are too irregular for the debris to be recognized as such). We have developed an image processing method to recognize very faint structures (Adaptive Laplace Filtering; see the last paragraph of the section), and applied it to a sample of 28 galaxies observed with the NTT (ESO), (Capaccioli et al. 1997).

"Double blobs": One of the most common type of structure (beside the small central disks) is what we call "double blobs" - two, more or less roundish, faint (1-10\% of the local surface brightness) enhancements of brightness; strictly symmetric relative to the galaxy center. We see them in almost all adaptive Laplace filtered $\mathrm{E}$ and $\mathrm{S} 0$ galaxies; in some cases even more than one pair in one galaxy, with different inclination to the major axis and of different scale lengths. As an example, NGC 1389 (Fig. 1) is shown with an outer, diffuse pair of blobs aligned with the major axis and with an inner, inclined pair of blobs (together with an edge on central disk).

\section{Adaptive Filtering:}

The problems with detection of faint structures in E type galaxies are:

- in the inner, bright parts: the steep gradient of the light distribution

- in the outer, faint parts: the low signal to noise ratio.

For both cases we need a filter which adapts itself to the local scale length of the signal (= adaptive filter). In the special case of structure detection, we use an adaptive filter of Laplacian type, i.e. essentially the second derivative of the light distribution (for details and the underlying theory see Lorenz et al. 1993). 

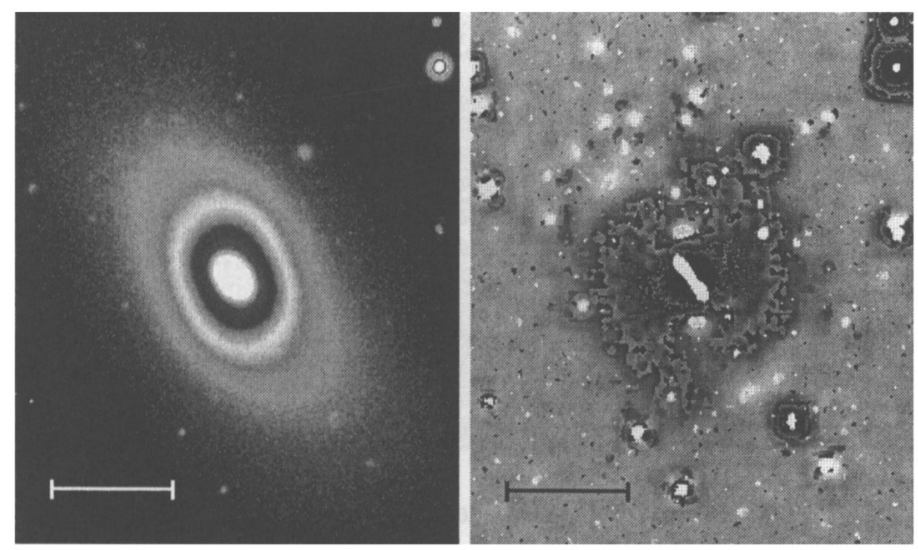

Figure 1. NGC 1389, I-Band (left) and adaptive Laplace filtered (right). The scale bars are $30^{\prime \prime}=2.5 \mathrm{kpc}$.

\section{Interpretation}

\section{Interpretation of "double blobs":}

There are two main phenomena which could provide the double blobs:

1. The bar: Only the ends of the bar are visible; the central part of the bar cannot be recognized against the bright center of the galaxy.

2. The ring, seen edge on: The brightness of the tangential regions of a ring is enhanced by the long line of sight inside of the ring.

There are two main arguments in favour of the second interpretation:

1. In case of bars, it is unlikely that we see always roundish structures. We should expect to see sometimes longer sections of bars which are elongated in the radial direction. Actually, we have found no such case. Instead, we see sometimes blobs elongated in tangential direction (e.g. Fig. 1, outer blobs). This is the direction in which the rings are broadened in the course of time by the precession of the orbits of the individual stars.

2. In some cases we see not only the tangential blobs, but longer sections of an inclined (not strictly edge on) ring (Fig. 2). Here we lose only the parts of rings, which are projected on the very inner bright region of the galaxy.

The double blobs are invisible on the colour index image of NGC 4767. Hence, they have nothing to do with ordinary (young and blue) spiral features.

\section{Some crude statistics:}

- If we assume a typical $10 \%$ of the local brightness for the double blobs, and they cover the order of $1 \%$ of the galaxy surface, they contribute about $10^{-3}$ of the galaxy light. Hence, we can assume for 1 ring $10^{-3}$ galaxy mass or $10^{8} M_{\odot}$, which is just a dwarf galaxy mass. 

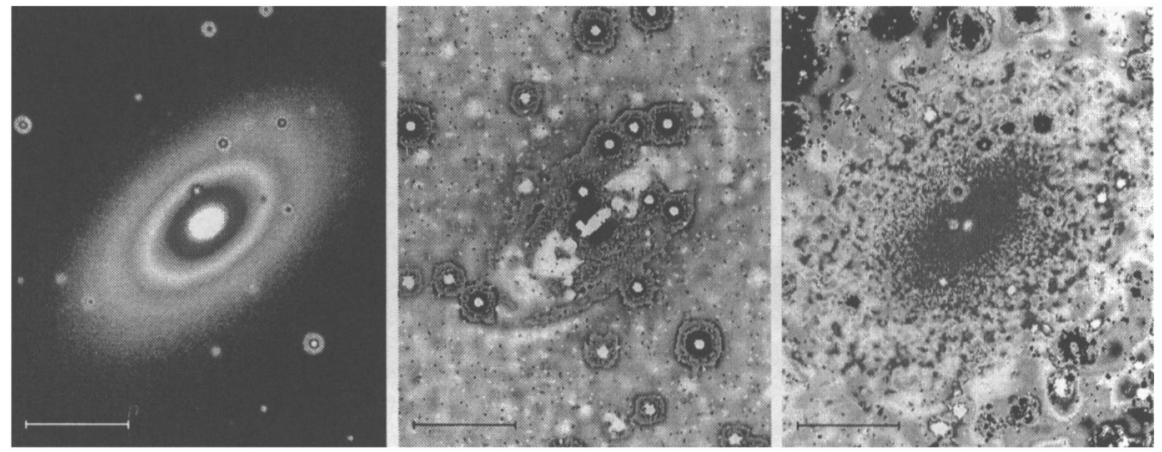

Figure 2. NGC 4767, $V$-Band (left), adaptive Laplace filtered (middle) and color index $V-I$ (right). The scale bars are $30^{\prime \prime}=6.75 \mathrm{kpc}$. The range of the false color code for $V-I$ covers $0.5 \mathrm{mag}$.

- If we see 1 ring per galaxy edge on, we must expect order of 10 similar rings which we do not see, because they are inclined to the line of sight and, thus, show less contrast.

- Hence, with a lifetime of a ring of $10^{8}$ years, we get formally $10^{11} M_{\odot}$ in 10 Gyr. Even if we take into account, that our estimates are very crude, this means: the assumed mechanism together with the observed quantities can easily provide a considerable fraction (say, up to $90 \%$ ) of a galaxy in a Hubble time. (Of course, for the mechanism to work, an initial "main body" must already exist.)

The entire sample can be accessed as colored images either in Richter et al. 1997, or on the World Wide Web (Böhm et al. 1997).

Acknowledgments. J. V. gratefully acknowledges financial support provided by the Academy of Finland and by the Estonian Science Foundation, Grant 2627. The images were processed with ESO MIDAS, where we have implemented the adaptive filter technique.

\section{References}

Barnes, J. E. 1992, A\&A, 393, 484

Böhm, P., Capaccioli, M. D’Onofrio, M., Longo, G., \& Richter, G.M. 1997, Atlas of Structures in Early Type Galaxies, http://www.aip.de/science/Atlas.html

Capaccioli, M., Longo, G., Böhm, P., Richter, G., \& D'Onofrio, M. 1997, Il Nuovo Cimento, 112, 243

Einasto, J., Kaasik, A., Kalamees, P., \& Vennik, J. 1975, A\&A, 40, 161

Helmi, A., White, S. D. M., de Zeeuw, P. T., Zhao, H. 1999, astro-ph/9911041

Johnston, K. V., Hernquist, L., \& Bolte, M. 1996, ApJ, 465, 278

Lorenz, H., Richter, G. M., Capaccioli, M., \& Longo, G. 1993, A\&A, 277, 321 
Richter, G., Capaccioli, M., Longo, G., Böhm, P., \& D'Onofrio, M. 1997, ASP Conf. Ser., 116, 480 